\title{
THE COMPLETE PADÉ TABLES OF CERTAIN SERIES OF SIMPLE FRACTIONS
}

\section{ALBERT EDREI}

Introduction. Let the expansion

$$
\sum_{k=0}^{\infty} a_{k} z^{k}=A(z) \quad\left(a_{0} \neq 0\right)
$$

have a positive radius of convergence and let its analytic continuation define a function $A(z)$, meromorphic in the whole plane. Put

$$
a_{-k}=0 \quad(k=1,2,3, \cdots)
$$

and associate with every pair $(m, n)$ of ordered, nonnegative integers the polynomial

$$
A_{m n}(z)=\left|\begin{array}{lllll}
1 & z & z^{2} & \cdots & z^{n} \\
a_{m+1} & a_{m} & a_{m-1} & \cdots & a_{m-n+1} \\
a_{m+2} & a_{m+1} & a_{m} & \cdots & a_{m-n+2} \\
\cdots & \cdots & \ldots & \cdots & \cdots \\
a_{m+n} & a_{m+n-1} & a_{m+n-2} & \cdots & a_{m}
\end{array}\right|\left(A_{m 0}(z) \equiv 1\right)
$$

and the Hankel determinant

$$
A_{m}^{(n)}=A_{m n}(0) \quad\left(A_{m}^{(0)}=1, m \geqq 0\right) .
$$

In everything that follows $\{m(\lambda)\}_{\lambda=1}^{\infty},\{n(\lambda)\}_{\lambda=1}^{\infty}$ denote two sequences of nonnegative integers such that

$$
A_{m}^{(n)} \neq 0 \quad(m=m(\lambda), n=n(\lambda) ; \lambda=1,2,3, \cdots),
$$

and we usually require

$$
m(\lambda) \rightarrow \infty, n(\lambda) \rightarrow \infty \quad(\lambda \rightarrow \infty) .
$$

We say that

$$
Q_{m n}(z)=\frac{A_{m n}(z)}{A_{m}^{(n)}} \quad(m=m(\lambda), n=n(\lambda))
$$

The research of the author was supported by a grant from the National Science Foundation GP-33175. 
is the normalized Pade denominator of the entry $(m, n)$ of the table of (1), and take for granted the existence and uniqueness of the corresponding normalized Padé numerator $P_{m n}(z)$; that is a polynomial of degree not greater than $m$ such that

$$
A(z) Q_{m n}(z)-P_{m n}(z)=z^{m+n+1} \delta(z),
$$

where $\delta(z)$ denotes a series of non-negative powers of $z$.

It is convenient to simplify the notations and, whenever there is no fear of confusion, to write

$$
A_{\lambda}(z)=A_{m n}(z), Q_{\lambda}(z)=Q_{m n}(z), P_{\lambda}(z)=P_{m n}(z)(m=m(\lambda), n=n(\lambda)) \text {. }
$$

Let the exact degrees of $Q_{\lambda}(z)$ and $P_{\lambda}(z)$ be respectively $d(\lambda)$ and $\delta(\lambda)$

$$
d=d(\lambda) \leqq n(\lambda), \quad \delta=\delta(\lambda) \leqq m(\lambda) .
$$

We now focus our attention on the factored forms

$$
Q_{\lambda}(z)=\prod_{j=1}^{d}\left(1-\frac{z}{\zeta_{j}}\right), \quad P_{\lambda}(z)=a_{0} \prod_{j=1}^{\delta}\left(1-\frac{z}{\omega_{j}}\right)
$$

with

$$
\begin{aligned}
\zeta_{j}=\zeta_{j}(\lambda) & (1 \leqq j \leqq d(\lambda)), \\
\omega_{j}=\omega_{j}(\lambda) & (1 \leqq j \leqq \delta(\lambda)) .
\end{aligned}
$$

We also need to introduce the sums

$$
\sigma_{k}(\lambda)=\sum_{j=1}^{d(\lambda)} \zeta_{j}^{-k}(\lambda), \quad \tau_{k}(\lambda)=\sum_{j=1}^{\delta(\lambda)} \omega_{j}^{-k}(\lambda),
$$

for $k=1,2,3, \cdots$, and the polynomials

$$
\begin{aligned}
& \left\{\begin{array}{l}
W_{\lambda}(z)=\frac{\sigma_{1}(\lambda)}{1} z+\frac{\sigma_{2}(\lambda)}{2} z^{2}+\cdots+\frac{\sigma_{s}(\lambda)}{s} z^{s} \quad(s \geqq 1), \\
W_{\lambda}(z) \equiv 0 \quad(s=0),
\end{array}\right. \\
& \begin{cases}W_{\lambda}(z)=\frac{\tau_{1}(\lambda)}{1} z+\frac{\tau_{2}(\lambda)}{2} z^{2}+\cdots+\frac{\tau_{s}(\lambda)}{s} z^{s} \quad(s \geqq 1), \\
W_{\lambda}(z) \equiv 0 \quad(s=0) .\end{cases}
\end{aligned}
$$

We propose to show the usefulness of the following 
Lemma A. Consider the expansion (1) of the meromorphic function $A(z)$ and let the sequences of integers $\{m(\lambda)\}_{\lambda}$ and $\{n(\lambda)\}_{\lambda}$ satisfy (4) and

$$
n(\lambda) \rightarrow \infty \quad(\lambda \rightarrow \infty) .
$$

Use the simplified notations described above and assume that the Padé denominators $Q_{\lambda}(z)$ satisfy the following condition: there exist constants $\xi>0, \chi>1$ such that

$$
\sum_{j=1}^{d(\lambda)}\left|\zeta_{j}(\lambda)\right|^{-\xi} \leqq \chi \quad(\lambda=1,2,3, \cdots)
$$

Let the degrees of the polynomials $W_{\lambda}(z)$ and $W_{\lambda}^{\prime}(z)$ be bounded by s:

$$
\begin{cases}s=[\xi] & (\xi \neq \text { integer }), \\ s=\xi-1 & (\xi=\text { integer }) .\end{cases}
$$

Then, for some suitable $\rho>0$, there are no zeros of $P_{\lambda}(z)$ in $|z| \leqq \rho, \lambda>$ $\lambda_{0}$, and each of the three sequences

$$
\begin{gathered}
\left\{Q_{\lambda}(z) \exp \left(W_{\lambda}(z)\right)\right\}_{\lambda=1}^{\infty}, \quad\left\{P_{\lambda}(z) \exp \left(W_{\lambda}(z)\right)\right\}_{\lambda=1}^{\infty}, \\
\left\{W_{\lambda}(z)-W_{\lambda}(z)\right\}_{\lambda=1}^{\infty},
\end{gathered}
$$

is uniformly bounded on every compact set $D$.

Moreover,

$$
\left\{Q_{\lambda}(z) A(z)-P_{\lambda}(z)\right\} \exp \left(W_{\lambda}(z)\right) \rightarrow 0,
$$

uniformly on every compact set $D_{1}$ which does not contain poles of $A(z)$.

The introduction of the condition (15) in the study of the convergence of sequences of polynomials goes back to a classical paper of Lindwart and Pólya [9, p. 297]; it is obviously connected with the special form of Weierstrass' primary factor of genus $s$ :

$$
\left\{\begin{array}{l}
E(u, s)=(1-u) \exp \left(u+\frac{u^{2}}{2}+\cdots+\frac{u^{s}}{s}\right) \quad(s \geqq 1) \\
E(u, 0)=(1-u)
\end{array}\right.
$$

From this point on we confine our attention to a class of meromorphic functions for which the Lindwart-Pólya condition is easily verified. 
Functions of Grommer type. We say that $\mathrm{A}(z)$ is of Grommer type if

(i) it is meromorphic and nonrational;

(ii) representable in the form

$$
\mathrm{A}(z)=\kappa+\sum_{j=1}^{\infty} \frac{r_{j}}{\beta_{j}-z}=\sum_{k=0}^{\infty} a_{k} z^{k}
$$

where all the $\beta$ 's are real; no $\beta$ is zero and

$$
\begin{gathered}
\beta_{j} \rightarrow \infty \quad(j \rightarrow \infty) ; \\
\kappa \geqq 0, \quad \frac{r_{j}}{\beta_{j}}>0 \quad(j=1,2,3, \cdots), \\
\sum_{j=1}^{\infty} \frac{r_{j}}{\beta_{j}}<+\infty .
\end{gathered}
$$

As an immediate consequence of the above definition, we observe that $A(z)$ and $A(-z)$ are simultaneously of Grommer type. This enables us to impose, without loss of generality, an additional

Normalization. All our functions of Grommer type have infinitely many poles on the positive axis.

Functions of Stieltjes type. A function of Grommer type is said to be of Stieltjes type if it satisfies the additional conditions

$$
\beta_{j}>0 \quad(j=1,2,3, \cdots) .
$$

The convergence theorem proved here depends on Lemma $\mathrm{A}$ and on some well known properties of orthogonal polynomials. The necessary facts are summarized in a preliminary result which I state as

THEOREM 1. Let $A(z)$ be a meromorphic function of Grommer type. I. Then

$$
A_{m}^{(n)} \neq 0 \quad(m \geqq 0, n \geqq 0),
$$

provided

$$
m+n=\text { odd integer. }
$$

If, in addition, $A(z)$ is of Stieltjes type, (25) holds unrestrictedly for all $m \geqq 0, n \geqq 0$.

All the following assertions require

$$
m \geqq n-1 \geqq 0 .
$$


II. The zeros of $A_{m n}(z)$ are real.

III. Let $\beta$ and $\beta^{\prime}$ denote two consecutive poles of $A(z)$ :

$$
A(\beta)=A\left(\beta^{\prime}\right)=\infty, A(x) \neq \infty \quad\left(\beta<x<\beta^{\prime}\right) .
$$

Then $A_{m n}(x)$ has at most one zero (necessarily simple if it exists) in the interval

$$
\beta<x \leqq \beta^{\prime} .
$$

If $A(z)$ has a first pole, say $\beta_{1}\left(\beta_{1}<\beta_{j}, j>1\right)$, then $A_{m n}(x)$ has at most one zero (necessarily simple if it exists) in the interval

$$
x \leqq \beta_{1} \text {. }
$$

IV. If $m+n$ is odd and if

$$
\beta<0<\beta^{\prime},
$$

then $A_{m n}(x)$ does not vanish in the interval

$$
\beta \leqq x \leqq \beta^{\prime} .
$$

V. If $A(z)$ is also of Stieltjes type, then $A_{m n}(x)$ does not vanish in the interval (29), regardless of the parity of $m+n$.

Combining Lemma A and Theorem 1, I prove my main convergence result:

THEOREM 2. Let $A(z)$ be of Grommer type and let there be some $\xi>0$ such that

$$
\sum_{j=1}^{\infty} \frac{1}{\left|\boldsymbol{\beta}_{j}\right|^{\xi}}=\chi<+\infty .
$$

I. Then the zeros of $A(z)$ form a real sequence $\left\{\alpha_{j}\right\}_{j=1}^{\infty}$ such that

$$
\sum_{j=1}^{\infty} \frac{1}{\left|\alpha_{j}\right|^{\xi}} \chi
$$

II. Let the integer $s$ be defined by (16) and let $E(u, s)$ denote the primary factor of genus $s$.

Put

$$
g(z)=\prod_{j=1}^{\infty} E\left(\frac{z}{\alpha_{j}}, s\right), \quad h(z)=\prod_{j=1}^{\infty} E\left(\frac{z}{\beta_{j}}, s\right) .
$$

Then 


$$
A(z)=a_{0} \exp (\Omega(z)) \frac{g(z)}{h(z)} \quad(\Omega(0)=0),
$$

where $\Omega(z)$ is a polynomial of degree not greater than $s$.

III. If the sequences $\{m(\lambda)\}_{\lambda},\{n(\lambda)\}_{\lambda}$ satisfy the conditions (5) as well as

$$
m(\lambda)+n(\lambda)=\text { odd integer } \quad(\lambda=1,2,3, \cdots),
$$

we have (with the notations (10), (11), (12), and (13))

$$
\begin{gathered}
Q_{\lambda}(z) \exp \left(W_{\lambda}(z)\right) \rightarrow h(z), \\
P_{\lambda}(z) \exp \left(W_{\lambda}(z)\right) \rightarrow a_{0} g(z), \\
\left\{W_{\lambda}(z)-W_{\lambda}(z)\right\} \rightarrow \Omega(z),
\end{gathered}
$$

uniformly on every compact set $D$.

IV. If $A(z)$ is also of Stieltjes type the relations (36), (37), (38) hold without the restriction (35).

The condition (35) has been imposed for simplicity. In view of the identities $[1 ;$ p. 14]

$$
Q_{m, n+1}(z)=Q_{m, n}(z)-z Q_{m-1, n}(z) \frac{A_{m+1}^{(n+1)} A_{m-1}^{(n)}}{A_{m}^{(n+1)} A_{m}^{(n)}},
$$

it is not difficult to study the entries for which

$$
m(\lambda)+n(\lambda)=\text { even integer. }
$$

The elementary study [in $\S 3$ ] of the zeros of $A(z)$ shows that these zeros are all real and simple. It suggests a renumbering of the poles $\beta_{j}$, of the corresponding $r_{j}$, and of the zeros $\alpha_{j}$, characterized by the inequalities

$$
\cdots<\alpha_{-1}<\beta_{-1}<\alpha_{0}<\beta_{0}<0<\beta_{1}<\alpha_{1}<\dot{\beta_{2}}<\alpha_{2}<\cdots .
$$

If, in the Grommer case, there is a first pole $\beta_{N}(N \leqq 0)$, there may or may not exist some preceding zero $\alpha_{N}$.

If there is no such zero we introduce the conventions

$$
\alpha_{N}=\infty, \quad \frac{1}{\alpha_{N}}=0 .
$$

With this modified notation, we clearly have

$$
\sum_{j}\left|\frac{1}{\beta_{j}{ }^{k}}-\frac{1}{\alpha_{j}{ }^{k}}\right|<+\infty \quad(k=1,2,3, \cdots)
$$


and we may therefore introduce the quantities

$$
\gamma_{k}=\sum_{j}\left(\frac{1}{\beta_{j}{ }^{k}}-\frac{1}{\alpha_{j}{ }^{k}}\right) \quad(k=1,2,3, \cdots) .
$$

I mention without proof four elementary facts which depend on (41) and (42). They disclose the very simple structure of functions of Grommer type.

I. The polynomial $\Omega(z)$ of $(34)$ is given by

$$
\Omega(z)=\sum_{k=1}^{s} \frac{\gamma_{k} z^{k}}{k} \quad(s \geqq 1) .
$$

II. The relation (41) with $k=1$ shows that

$$
\prod_{j}\left(\frac{1-\frac{z}{\alpha_{j}}}{1-\frac{z}{\beta_{j}}}\right)=\prod(z),
$$

converges uniformly on every compact set $D_{1}$ which omits the poles of $A(z)$.

III. We always have

$$
A(z)=a_{0} \prod(z)
$$

IV. The relations (41), (43) and (44) do not depend on the condition (31). They are valid for all functions of Grommer type (including those of infinite order).

The properties II, III and IV were noticed and pointed out to me by my friend Wolfgang Jurkat.

Among the simplest functions to which Theorem 2 may be applied I mention a function of Stieltjes type:

(i) $\frac{\Gamma(b-z)}{\Gamma(a-z)}=\frac{\Gamma(b)}{\Gamma(a)} \prod_{n=0}^{\infty}\left(\frac{1-\frac{z}{n+a}}{1-\frac{z}{n+b}}\right) \quad(0<b<a<b+1)$,

and a function of Grommer type: 
More generally we may consider, instead of (ii),

$$
\frac{\Psi(z)}{z}
$$

where $\Psi(z)$ is a meromorphic function of finite order satisfying all the following conditions:

I. $\Psi(z)$ is real, periodic, with a real period and $\Psi(0)=0, \Psi^{\prime}(0)>0$.

II. The zeros and poles of $\Psi(z)$ are all real, simple and interlaced. [This means that between any two consecutive poles of $\Psi(z)$ there is exactly one zero.]

I have studied elsewhere [5] the Padé tables of the functions

$$
(\cos z)^{\mu},\left(\frac{\sin z}{z}\right)^{\mu} \quad(\mu=\text { integer }), \quad\left(\frac{\tan z}{z}\right)^{2} .
$$

As far as I can see, the functions (45) cannot be treated by the methods of the present note.

Lemma A seems to me of some independent interest and I therefore propose to give elsewhere some extensions and applications of this result. In particular, I shall replace the Lindwart-Pólya condition by a more general one which does not preclude the appearance of functions of infinite order. This extended form of Lemma A leads to an extension of Theorem 2 which is no longer restricted by (31) and therefore covers all functions of Grommer type.

1. Connections with the moment problem and with some recent publications. The relations (21), (22), and (23) show that the coefficients $a_{k}$ form a sequence of Stieltjes moments. We then say, with Baker [2], that the generating function $\mathrm{A}(\boldsymbol{z})$ is a series of Stieltjes. Statement and proof of Theorem 2 depend essentially on one additional property: $\mathrm{A}(z)$ is a meromorphic function of finite genus.

A general series of Stieltjes need not be meromorphic and if it is meromorphic it may have any order (and therefore any genus) finite or $\infty$. This accounts for the fact that Theorem 1 is not contained in the results of Baker [2; pp. 8-19].

An anonymous referee has drawn my attention to two notes of Franzen [6], [7], which, like the present one, are concerned with convergence of the Padé table of a series of simple fractions. In view of the identity of subject matter, one could fear a certain amount of overlapping of methods or results.

As far as I can see

(i) there is no overlapping of results and my assertions are not implied by those of Franzen; 
(ii) the content of the major part of Franzen's Lemma $1[6$, p. 257] only differs in notation from an identity [3, Chapter 5] which I applied systematically in a paper published in 1940. It is not unlikely that the identity in question has been known to, and used by, mathematicians of an earlier age.

2. Notations. Beside $A(z)$ we consider series

$$
B(z)=\sum_{k=0}^{\infty} b_{k} z^{k}, \quad D(z)=\sum_{k=0}^{\infty} d_{k} z^{k}, \cdots .
$$

Expressions such as

$$
B_{m n}(z), \quad B_{m}^{(n)}, D_{m n}(z), \quad D_{m}^{(n)} ; \cdots
$$

are obtained by replacing in (3) and (4), the $a$ 's by $b$ 's, $d$ 's, $\cdots$.

We always take

$$
0=a_{-k}=b_{-k}=d_{-k}=\cdots \quad(k=1,2,3, \cdots) .
$$

Determinants of order zero are, by definition, equal to 1 .

It is convenient to consider beside $A_{m}^{(n)}, B_{m}^{(n)}, D_{m}^{(n)}, \ldots$ determinants such as

$$
\mathcal{A}_{k}^{(n)}=(-1)^{(n(n-1)) / 2} A_{m}^{(n)}, \mathcal{B}_{k}^{(n)}, D_{k}^{(n)}, \cdots
$$

with

$$
k=m-n+1
$$

It is clear that $A_{k}^{(n)}$ is the determinant obtained by reversing the order of the columns of $A_{m}^{(n)}$; the integer $k$ is the suffix of the entry in the upper left corner of $\mathcal{A}_{k}^{(n)}$.

Throughout the paper $\delta(z)$ denotes a series of non-negative powers of $z$, not necessarily the same one at each occurrence.

All the above notational conventions are taken for granted; we shall use them without reminding the reader of the meaning of the symbols.

3. Proof of assertions I and II of Theorem 2. Let

$$
z=r e^{i \theta} \quad r \geqq 0, \quad 0 \leqq \theta \leqq \pi .
$$

From (21) and (3.1) we deduce

$$
|A(z)| \leqq \kappa+\frac{1}{\min (\sin \theta / 2, \cos \theta / 2)} \sum_{j=1}^{\infty} \frac{\left|r_{j}\right|}{\left|\beta_{j}\right|+r} .
$$


We take for granted elementary facts of the theory of entire and meromorphic functions and use the classical notations of Nevanlinna. [What little we need is adequately covered in [12; pp. 246-254 and pp. $284 \mathrm{~b}-284 \mathrm{~g}$ ] ]

From (3.2) it is immediately deduced that Nevanlinna's mean $m(r, A)$ remains bounded:

$$
m(r, A)=0(1) \quad(r \rightarrow \infty) .
$$

To study the zeros $\left\{\alpha_{j}\right\}_{j}$ of $A(z)$ it is convenient to introduce the variable $t=z^{-1}$. This leads to

$$
L(t)=\frac{1}{t} A\left(\frac{1}{t}\right)=\frac{\kappa}{t}+\sum_{j=1}^{\infty} \frac{\rho_{j}}{t-\left(1 / \beta_{j}\right)},
$$

with

$$
0<\rho_{j}=\frac{r_{j}}{\beta_{j}}, \quad \sum_{j=1}^{\infty} \rho_{j}<+\infty,
$$

and, for real values of $t$,

$$
L^{\prime}(t)<0 \quad(t \neq 0) .
$$

From (3.4) and (3.5) it follows immediately that all the zeros of $L(t)$ are real and simple.

Now return to $A(z)$ and let $\beta, \beta^{\prime}\left(\beta<\beta^{\prime}\right)$ be two consecutive poles of $A(z)$.

The observations concerning the zeros of $L(t)$ clearly imply the following:

(i) if $\beta<0<\beta^{\prime}$ then

$$
A(x)>0 \quad\left(\beta<x<\beta^{\prime}\right) ;
$$

(ii) in every other open interval between two consecutive poles of $A(z)$ there is a single (simple) zero $\alpha$, of $A(z)$;

(iii) if $A(z)$ has a first pole $\beta_{1}$, there is at most one zero of $A(z)$ in the interval $\left(-\infty, \beta_{1}\right)$.

By assumption, $A(0) \neq 0$, so that the preceding remarks show that, for every $\tau>0$,

$$
\sum_{j=1}^{\infty}\left|\alpha_{j}\right|^{-\tau}, \quad \sum_{j=1}^{\infty}\left|\beta_{j}\right|^{-\tau}
$$

converge or diverge simultaneously. 
We have as yet made no use of the assumption (31). Hence, up to this point, the arguments of the present section apply to all functions of Grommer type.

If (31) holds, it is clear that (32) follows. We have thus proved assertion I of Theorem 2.

Now defining $s$ by the relations (16), we deduce from the elementary theory of entire functions that the products (33) are convergent and that $A(z)$ is of the form (34) with $\Omega(z)$ entire.

To verify that $\Omega(z)$ is a polynomial of degree $\leqq s$, we observe that

$$
m(r, h)=o\left(r^{s+1}\right), \quad m(r, 1 / g)=m(r, g)+0(1)=o\left(r^{s+1}\right) .
$$

[The first relation in (3.6) follows from (31), (16), and the well known behavior of canonical products [10; pp. 226-227]; the result concerning $m(r, 1 / g)$ requires, in addition, the use of (32) and of Nevanlinna's first fundamental theorem [10, p. 168]].

We now use (34) (without assuming that $\Omega(z)$ is a polynomial) as well as (3.3) and (3.6). This leads us to

$m\left(r, \exp (\Omega(z)) \leqq m\left(r, A / a_{0}\right)+m(r, h)+m(r, 1 / g)=o\left(r^{s+1}\right) \quad(r \rightarrow \infty)\right.$,

which is only possible if $\Omega(z)$ is a polynomial of degree not exceeding $s$.

The proof of assertion II of Theorem 2 is now complete.

4. Orthogonality relations. Let $A(z)$ be representable in the form (21). In order to apply classical results of the theory of orthogonal polynomials, it is convenient to introduce the notations

$$
\begin{aligned}
& t_{j}=\frac{1}{\beta_{j}}, \quad t_{\infty}=0 \quad\left(t_{\infty}{ }^{0}=1\right), \\
& \rho_{j}=\frac{r_{j}}{\beta_{j}}>0, \quad \rho_{\infty}=\kappa \geqq 0 .
\end{aligned}
$$

We assume that $A(z)$ is of Grommer type and that $\beta_{j}<0$, for some $j$. If $A(z)$ is of Stieltjes type the notation requires minor changes and some additional statements are possible; the details are obvious and will be left to the reader.

Throughout this section we number the $\beta$ 's as well as the corresponding $t$ 's and $\rho$ 's as indicated in (39).

Introduce a sequence of functions

$$
\Psi_{k}(t) \quad(k=0,1,2,3, \cdots),
$$

of bounded variation in $\left[t_{0}, t_{1}\right]$, by the conditions 


$$
\begin{gathered}
\Psi_{k}\left(t_{0}\right)=0, \\
\Psi_{k}(t)=\sum_{t_{l} \leqq t} \rho_{j} t_{j}^{k} \quad\left(t>t_{0}\right) .
\end{gathered}
$$

It is immediately clear that

$$
d \Psi_{k}(t)=t d \Psi_{k-1}(t) \quad(k \geqq 1),
$$

and that:

For $k=2 \ell(\ell=0,1,2,3, \cdots)$ each $\Psi_{k}(t)$ is a nondecreasing function.

We also note that in view of the definition of the Riemann-Stieltjes integral

$$
A(z)=\int_{t_{0}}^{t_{1}} \frac{d \Psi(t)}{1-t z}=\sum_{j=0}^{\infty} a_{j} z^{j} \quad\left(\Psi(t) \equiv \Psi_{0}(t)\right),
$$

and, more generally, since

$$
a_{j+k}=\int_{t_{0}}^{t_{1}} t^{j+k} d \Psi_{0}(t)=\int_{t_{0}}^{t_{1}} t j d \Psi_{k}(t) \quad(j \geqq 0, k \geqq 0),
$$

we find

$$
\int_{t_{0}}^{t_{1}} \frac{d \Psi_{k}(t)}{1-t z}=\sum_{j=0}^{\infty} a_{j+k} z^{j}
$$

For every fixed $k \geqq 0$, we introduce the sequence of polynomials

$\varphi_{n}(z ; k)=\left|\begin{array}{ccccc}a_{k} & a_{k+1} & a_{k+2} & \cdots & a_{k+n} \\ a_{k+1} & a_{k+2} & a_{k+3} & \cdots & a_{k+n+1} \\ \cdots & \cdots & \cdots & \cdots & \cdots \\ a_{k+n-1} & a_{k+n} & a_{k+n+1} & \cdots & a_{k+2 n-1} \\ 1 & z & z^{2} & \cdots & z^{n}\end{array}\right| \quad(n=1,2,3, \cdots)$

Classical fundamental relations, valid for $k \geqq 0, n \geqq 1$, are immediately deduced from the explicit form (4.3) and may be written as

$$
\int_{t_{0}}^{t_{1}} \varphi_{n}(t ; k) t^{j} d \Psi_{k}(t)=0 \quad(j=0,1,2, \cdots, n-1),
$$

and

$$
\int_{t_{0}}^{t_{1}} \varphi_{n}(t ; k) t^{n} d \Psi_{k}(t)=\mathcal{A}_{k}^{(n+1)}
$$


If $k$ is even we deduce from (4.3), (4.4) and (4.5)

$$
\mathcal{A}_{1}^{(n)} \mathcal{A}_{k}^{(n+1)}=\int_{t_{0}}^{t_{1}} \varphi_{n}^{2}(t ; k) d \Psi_{k}(t)>0
$$

and, since by (4.2) $a_{k}=A_{k}^{(1)}>0$, an obvious induction and (4.6) yield

$$
A_{k}^{(n)}>0 \quad(k=2 \ell, n=1,2,3, \cdots) .
$$

The relations (4.4), (4.5), (4.6) and (4.7) show that, for $k$ fixed and even, the sequence of polynomials

$$
\left\{\varphi_{n}(z ; k)\right\}_{n=1}^{\infty},
$$

is orthogonal in the usual sense.

For $k=2 \ell+1,(4.6)$ and (4.7) need not hold. However (4.4) and (4.5) are unaffected and we may consequently extend, with few changes, many proofs based on the orthogonality of the sequence (4.8).

These remarks lead to

LEMмa 4.1. The polynomials in (4.8) have the following properties.

I. Their zeros are all real and simple. If $k$ is even they are located in the open interval $\left(t_{0}, t_{1}\right)$ and the degree of $\varphi_{n}$ is exactly $n$.

If $k$ is odd, the degree of $\varphi_{n}$ is at least $n-1$. Moreover, if $\varphi_{n}$ is of degree $n$ it has at most one zero outside $\left(t_{0}, t_{1}\right)$; if it is of degree $n-1$ all its zeros lie in $\left(t_{0}, t_{1}\right)$.

II. Consider the intervals

$$
J_{j}: t_{j+1} \leqq t<t_{j},
$$

and, if $\mathrm{A}(z)$ has a first pole $\boldsymbol{\beta}_{-N}(N \geqq 0)$, also

$$
J_{\infty}: t_{-N} \leqq t<t_{\infty} \quad\left(t_{\infty}=0\right) .
$$

Then $\varphi_{n}(z ; k)$ has at most one zero in each interval $J_{j}\left(\right.$ or $\left.J_{\infty}\right)$.

Proof. For $k$ even, the sequence in (4.8) is orthogonal and consequently:

(i) assertion I coincides with a classical result [11, p. 44];

(ii) assertion II follows immediately from [11, p. 50, Theorem 3.41.2] .

For $k$ odd, the interested reader will find it easy to adapt the proofs in [11]. It should however be observed that our statements of Lemma 4.1 and Theorem 1 are more general than necessary to obtain Theorem 2: in Grommer's case, the only polynomials $A_{m n}(z)$ which we consider correspond to polynomials $\varphi_{n}(z ; 2 \ell)$. In Stieltjes' case the sequence (4.8) is orthogonal for all integers $k \geqq 0$. 
5. The zeros of $A_{m n}(z)$. A comparison of (4.3) and (3) shows that

$$
z^{n} \varphi_{n}(1 / z ; k)=(-1)^{(n(n-1)) / 2} A_{m n}(z),
$$

with

$$
m=k+n-1 \geqq n-1 \geqq 0 .
$$

In particular the conditions

$$
m \geqq n-1, \quad m+n=\text { odd integer, }
$$

imply that $k$ is even and non-negative.

Assertions II, III and IV of Theorem 1 follow immediately from Lemma 4.1, (4.1), (5.1) and (5.2).

If $A(z)$ is of Stieltjes type, the orthogonality of the sequence (4.8) does not depend on the parity of the (fixed) integer $k$. Hence assertion $V$ follows readily from assertion I of Lemma 4.1.

6. Hankel determinants of functions of Grommer type.

Lemma 6.1. Assume that $\mathrm{A}(z)$ is of Grommer type. Then

$$
\frac{1}{A(z)}=b_{0}+b_{1} z-z^{2} D(z)=\sum_{k=0}^{\infty} b_{k} z^{k}
$$

where

$$
D(z)=\sum_{k=0}^{\infty} d_{k} z^{k}
$$

is also of Grommer type.

We always have

$$
A_{n}^{(n)} \neq 0 \quad(m \geqq 0, n \geqq 0, m+n=2 \ell+1, \ell=, 0,1,2,3, \cdots) .
$$

Proof. By definition

$$
\mathcal{A}_{k}^{(n)}=(-1)^{n(n-1) / 2} A_{k+n-1}^{(n)} \quad(k \geqq 0, n \geqq 1) .
$$

In particular, consider (6.4) with $k=0$ and use the identity [ 1 ; p. 8, formula (1.7)]

$$
B_{n}^{(m)}=(-1)^{m n} a_{0}{ }^{-m-n} A_{m}^{(n)} \quad(m \geqq 0, n \geqq 0)
$$

connecting the Hankel determinants of any two series $A(z), B(z)$ such that $A(z) B(z) \equiv 1 \quad\left(a_{0}=a(0) \neq 0\right)$. We find, in view of $(6.1)$, 


$$
\begin{aligned}
A_{0}^{(n)} & =(-1)^{(n(n-1)) / 2} A_{n-1}^{(n)}=(-1)^{(n(n-1)) / 2} a_{0}^{2 n-1} B_{n}^{(n-1)} \\
& =(-1)^{(n(n-1) / 2)+n-1} D_{n-2}^{(n-1)} a_{0}{ }^{2 n-1}=a_{0}{ }^{2 n-1} \mathcal{D}_{0}^{(n-1)} \quad(n \geqq 2),
\end{aligned}
$$

and hence $D_{0}^{(n)}>0 \quad(n \geqq 0)$. By Grommer's fundamental result $[8$, p. 154, Satz I] the preceding inequalities imply that $D(z)$ is of Grommer type. Then (4.7), with $A$ replaced by $D$, yields

$$
\begin{aligned}
0<D_{k}^{(n)}= & (-1)^{(n(n-1)) / 2} D_{k+n-1}^{(n)}=(-1)^{(n(n-1)) / 2+n} B_{k+n+1}^{(n)} \\
& (n \geqq 1, k=2 \ell, \ell=0,1,2,3, \cdots) .
\end{aligned}
$$

Using (6.5) once more, we find

$$
A_{n}^{(k+n+1)} \neq 0 \quad(n \geqq 1, k=2 \ell, \ell=0,1,2,3, \cdots) .
$$

Trivially

$$
A_{0}^{(k+1)}=a_{0}^{k+1}>0,
$$

so that (6.8) remains valid for $n=0$.

From (4.7) and (6.4) we also deduce

$$
A_{k+n-1}^{(n)} \neq 0 \quad(n \geqq 1, k=2 \ell, \ell=0,1,2,3, \cdots) .
$$

The relations (6.3) are obvious consequences of (6.8), (6.9) and (6.10).

The proof of Lemma 6.1 is now complete. In the special case of functions of Stieltjes type it is possible to provide additional information.

LemMa 6.2. If $A(z)$ is of Stieltjes type, then

$$
\frac{1}{A(z)}=b_{0}-z F(z)
$$

where $F(z)$ is also of Stieltjes type.

We always have

$$
\mathcal{A}_{k}^{(n)}>0 \quad(n \geqq 0, k \geqq 0)
$$

and more generally

$$
A_{m}^{(n)} \neq 0 \quad(m \geqq 0, n \geqq 0) .
$$

Proof. The inequalities (6.12) state the fact that, for functions of Stieltjes type, the inequalities (4.7) also hold for odd values of $k$ (because of the unrestricted orthogonality of the sequences (4.8)).

Our definition of $F(z)$ implies

$$
f_{k}=-b_{k+1} \quad(k=0,1,2,3, \cdots) .
$$


We use (6.5), as in the proof of (6.6) and take (6.14) into account; this leads us to

$$
\begin{aligned}
A_{1}^{(n)} & =(-1)^{(n(n-1) / 2} A_{n}^{(n)}=(-1)^{n(n-1) / 2+n} a_{0}^{2 n} B_{n}^{(n)} \\
& =(-1)^{n(n-1) / 2} a_{0}^{2 n} F_{n-1}^{(n)}=a_{0}^{2 n} \Im_{0}^{(n)}>0 \quad(n \geqq 1) .
\end{aligned}
$$

Hence, by Grommer's theorem, $F(z)$ is of Grommer type. Since $A(z)$ is of Stieltjes type, the results of $\S 3$ show that all the zeros of $A(z)$ are real and lie in the interval

$$
\beta_{1}<x \quad\left(\beta_{1}>0\right) .
$$

Returning to (6.11) we conclude that all the poles of $F(z)$ lie in the interval (6.15) and consequently, $F(z)$ which is of Grommer type, must also be of Stieltjes type. Hence (6.12) (already proved) holds wit A replaced by 7 :

$$
\Im_{k}^{(n)}>0 \quad(n \geqq 0, k \geqq 0) .
$$

The relations (6.13) follow from (6.12) and (6.16) exactly like (6.3) follows from (4.7) and (6.7).

Remark. Grommer's theorem is the deepest result used in the present note. We only need it to show that $D(z)$ in $(6.1)$ and $F(z)$ in (6.11) are both of Grommer type.

A completely elementary proof of these facts (independent of Grommer's theorem) readily follows from the study of the decomposition in simple fractions of the rational functions

$$
\left(\kappa+\sum_{j=1}^{N} \frac{r_{j}}{\beta_{j}-z}\right)^{-1} \quad(N=1,2,3, \cdots) .
$$

7. Proof of Theorem 1. Assertion I is immediately deduced from (6.3) and (6.17).

All other assertions of Theorem 1 have been established in $\S 5$.

8. Estimates used in the proof of Lemma $A$. Let $A(z)$ be meromorphic in the whole plane and let (1) be its Taylor expansion. [ It is not assumed that $A(z)$ is of Grommer type.]

Let $\mathcal{N}(t, \lambda)$ denote the number of zeros of $Q_{\lambda}(z)$ in the disk $|z| \leqq t$. From (15) we deduce that the bound

$$
\mathcal{N}(t, \lambda) \leqq \chi t^{\xi} \quad(0 \leqq t)
$$

holds uniformly for all $\lambda$. In particular there are no zeros of any $Q_{\lambda}(z)$ in the disk

$$
|z|<\chi^{-1 / \xi}=\rho^{\prime} .
$$


Lemma 8.1. Let $R>1$ be given, let $W_{\lambda}(z)$ be defined by (12). Then (8.3)

$$
\left|Q_{\lambda}(z) \exp \left(W_{\lambda}(z)\right)\right| \leqq \exp \left(K_{2} \chi|z|^{\xi}\right) \quad\left(0<K_{2}=K_{2}(s, \xi)=\text { const. }\right),
$$

$$
\left|W_{\lambda}(z)\right| \leqq(1+\chi) n^{1-(1 / 5)} s\left(1+|z|^{s}\right) \quad(s \geqq 1, n=n(\lambda)) .
$$

Both (8.3) and (8.4) hold uniformly for all $z$ and all $\lambda$.

With

$$
|z| \leqq \rho^{\prime \prime}=\rho^{\prime} / 2
$$

and $\rho$ ' defined by (8.2), we also have

$$
\left|Q_{\lambda}(z) \exp \left(W_{\lambda}(z)\right)\right| \geqq \exp \left(-\frac{2 x|z|^{\xi}}{s+1}\right) \quad(s \geqq 0),
$$

uniformly in $\lambda$.

PRoof. For $|u| \leqq 1 / 2$, the primary factor satisfies the elementary estimate

$$
|\log | E\left(u, s|| \leqq \frac{2}{s+1}|u|^{s+1} \leqq \frac{2}{s+1}|u|^{s}\right.
$$

By (8.2) and (8.5), for every zero $\zeta_{j}(\lambda)$ of $Q_{\lambda}(z)$,

$$
\left|\frac{z}{\zeta_{j}(\lambda)}\right| \leqq \frac{1}{2}
$$

and hence (8.6) follows from (8.7).

A slightly different treatment of the primary factor $[10$, p. 225 , formulae (9) and (10)] yields

$$
\begin{aligned}
& \log |E(u, s)| \leqq K_{1} \frac{|u|^{s+1}}{1+|u|} \quad(s \geqq 1), \\
& \log |E(u, 0)| \leqq \log (1+|u|),
\end{aligned}
$$

with $\quad K_{1}=K_{1}(s)=3 e(2+\log s)$. Since $0 \leqq s+1-\xi<1$, implies, for all $u$,

$$
\log |E(u, s)| \leqq K_{2}|u|^{\xi},
$$

with $K_{2}=K_{1}(s)(s \geqq 1)$ [for $s=0, K_{2}$ is a function of $\xi>0$ ].

The inequality (8.3) follows from (8.9) and (15).

To prove (8.4), denote by $k$ an integer such that $1 \leqq k \leqq s$. Then, by (16),

$$
\frac{\xi}{k} \geqq \frac{\xi}{s}>1 .
$$


From Hölder's inequality, (11), (15), and (8.10), we deduce

$$
\begin{aligned}
\sigma_{k}(\lambda) & \leqq\left\{\sum_{j=1}^{d(\lambda)}\left|\zeta_{j}(\lambda)\right|^{-\xi}\right\}^{k / \xi}\{d(\lambda)\}^{1-k / \xi} \\
& \leqq \chi^{k / \xi} n^{1-k / \xi} \leqq(1+\chi) n^{1-1 / \xi} \quad(1 \leqq k \leqq s)
\end{aligned}
$$

and (8.4) follows. The proof of Lemma 8.1 is now complete.

9. Proof of Lemma A. Take $R>1$, large enough to imply

$$
\triangle \subset\{z:|z| \leqq R\}=\Delta(R) ; D_{1} \subset \Delta(R) ;
$$

it is no restriction to assume

$$
A\left(R e^{i \theta}\right) \neq \infty \quad(0 \leqq \theta<2 \pi) .
$$

Let the poles of $A(z)$ be

$$
b_{1}, b_{2}, b_{3}, \cdots, b_{N}, \cdots
$$

[Multiple poles appear in (9.3) as often as indicated by their multiplicities.] Put

$$
Y(z)=\prod_{j=1}^{N}\left(1-\frac{z}{b_{j}}\right)
$$

and assume that the sequence (9.3) has been arranged so that $A(z) Y(z)$ is regular for $z \in \Delta(2 R)$. Choose $M_{1}$ such that

$$
\max _{|z| \leqq 2 R}|A(z) Y(z)| \leqq M_{1} .
$$

In view of $(9.2)$ no $|b|$ is equal to $R$ and hence

$$
\min _{|z|=R}|Y(z)|=\mu>0 .
$$

Since $\supset_{1}$ is compact, and contains no zeros of $Y(z)$, we also have

$$
\min _{z \in D_{1}}|Y(z)|=\mu^{\prime}>0 .
$$

We now select a contour of integration

$$
\mathcal{G}: w=2 R e^{i \theta} \quad(0 \leqq \theta<2 \pi)
$$

and take

$$
z \in \Delta(R), \quad m=m(\lambda), n=n(\lambda) .
$$


We now follow a method used in $[4, \S 2]$ : from (7) and Cauchy's formula we deduce

$$
A(z) Y(z) Q_{\lambda}(z)-Y(z) P_{\lambda}(z)=
$$

$$
\frac{z^{m+n+1}}{2 \pi i} \int_{\mathcal{G}} \frac{A(w) Y(w) Q_{\lambda}(w)}{w^{m+n+1}(w-z)} d w=I(z)
$$

The omission in the above integral of a term involving $Y(w) P_{\lambda}(w)$ is justified by the remark that degree $\left\{Y(z) P_{\lambda}(z)\right\} \leqq N+m \leqq n(\lambda)+$ $m(\lambda)$. In view of $(14)$, the latter inequality holds for $\lambda>\lambda_{0}$.

Multiply the three members of $(9.8)$ by $\exp \left(W_{\lambda}(z)\right)$ and use (9.4), (8.3) and (8.4). This leads to

$$
\begin{aligned}
& \max _{|z| \leqq R}\left|I(z) \exp \left(W_{\lambda}(z)\right)\right| \leqq \\
& M_{1} 2^{-m} \exp \left(K_{2} X(2 R)^{\xi}\right) \exp \left(2(1+\chi) n^{1-(1 / \xi)} s\left(1+(2 R)^{s}\right)-n \log 2\right) .
\end{aligned}
$$

In view of (9.1), (9.6) and (9.8) we deduce from (9.9)

$$
\begin{aligned}
\max _{z \in D_{1}}\left|\left(A(z) Q_{\lambda}(z)-P_{\lambda}(z)\right) \exp \left(W_{\lambda}(z)\right)\right| & \\
\leqq & \frac{M_{1}}{\mu^{\prime}} \exp \left(K_{2} X(2 R)^{\xi}\right) \exp \left(\frac{-n(\lambda) \log 2}{2}\right) \quad\left(\lambda>\lambda_{0}\right),
\end{aligned}
$$

and (19) follows.

From (9.8), (9.9), (9.4) and (8.3) we also deduce

$$
\begin{aligned}
& \max _{|z| \leqq R}\left|Y(z) P_{\lambda}(z) \exp \left(W_{\lambda}(z)\right)\right| \\
& \leqq 2 M_{1} \exp \left(K_{2} X(2 R)^{\xi}\right)=K_{3}(R) \quad\left(\lambda>\lambda_{0}\right),
\end{aligned}
$$

and by (9.5) this implies

$$
\max _{|z|=R}\left|P_{\lambda}(z) \exp \left(W_{\lambda}(z)\right)\right| \leqq \frac{K_{3}}{\mu} \quad\left(\lambda>\lambda_{0}\right) .
$$

By the maximum modulus principle we replace in the above inequality $\max _{|z|=R}$ by $\max _{|z| \leqq R}$ and thus verify that the second sequence in (17) is uniformly bounded on $\Delta(R) \supset D$. The behavior of the first sequence in (17) is already known from (8.3) and there only remains to study (18).

Combining (8.6) with (19) (which is already proved) we deduce the existence of $\epsilon^{\prime}>0$ and $\rho\left(0<\rho \leqq \rho^{\prime \prime}\right)$ such that 


$$
\begin{gathered}
\left|A(z) Q_{\lambda} \exp \left(W_{\lambda}(z)\right)\right| \geqq 2 \epsilon^{\prime} \quad(|z| \leqq \rho), \\
\epsilon^{\prime} \leqq\left|P_{\lambda}(z) \exp \left(W_{\lambda}(z)\right)\right| \quad\left(|z| \leqq \rho, \lambda>\lambda_{0}\right) .
\end{gathered}
$$

In view of the boundedness of the second sequence in (17) we also have, for some suitable bound $M^{\prime}\left(0<M^{\prime}<+\infty\right)$

$$
\left|P_{\lambda}(z) \exp \left(W_{\lambda}(z)\right)\right| \leqq M^{\prime} \quad\left(|z| \leqq \rho^{\prime}\right)
$$

It follows from $(9.10)$ that the polynomials $\left\{P_{\lambda}(z)\right\}_{\lambda}\left(\lambda>\lambda_{0}\right)$ have no zeros in $|z| \leqq \rho$. Hence, returning to the factored form of $P_{\lambda}(z)$ in (10), we find

$$
\rho<\left|\omega_{j}(\lambda)\right| \quad\left(\lambda>\lambda_{0}, j=1,2,3, \cdots, \delta(\lambda)\right) .
$$

In particular, for $|z| \leqq \rho$, we have, using the notations (11) and (13),

$$
\log \left(\frac{P_{\lambda}(z)}{a_{0}}\right)=-\mathcal{W}_{\lambda}(z)-\sum_{k=s+1}^{\infty} \frac{\tau_{k}(\lambda)}{k} z^{k} \quad(s \geqq 1),
$$

Consider now

$$
\log \left(\frac{P_{\lambda}(z)}{a_{0}}\right)+W_{\lambda}(z)=W_{\lambda}(z)-W_{\lambda}^{\prime}(z)-\sum_{k=s+1}^{\infty} \frac{\tau_{k}(\lambda)}{k} z^{k}
$$

and notice that (9.11) implies

$$
\operatorname{Re}\left\{\log \left(\frac{P_{\lambda}(z)}{a_{0}}\right)+W_{\lambda}(z)\right\} \leqq \log \frac{M^{\prime}}{\left|a_{0}\right|} \quad(|z| \leqq \rho) .
$$

Hence by a classical result analogous to Cauchy's inequality [ 12 , p. 86], (9.12) and (9.13) yield

$$
\rho^{k}\left|\frac{\sigma_{k}(\lambda)-\tau_{k}(\lambda)}{k}\right| \leqq 4 \log \left(M^{\prime} /\left|a_{0}\right|\right) \quad(k=1,2,3, \cdots, s),
$$

and therefore

$$
\max _{|z| \leqq R}\left|W_{\lambda}(z)-\eta_{\lambda}(z)\right| \leqq 4 \log \frac{M^{\prime}}{\left|a_{0}\right|} \sum_{k=1}^{s}\left(\frac{R}{\rho}\right)^{k}
$$

This proves the uniform boundedness of the sequence (18) and the proof of Lemma $\mathrm{A}$ is complete.

10. Proof of assertions III and IV of Theorem 2 in the case $m \geqq$ $n-1$. We assume

$$
(10.1) m(\lambda) \geqq n(\lambda)-1 \quad(\lambda=1,2,3, \cdots) ; n(\lambda) \rightarrow \infty(\lambda \rightarrow \infty),
$$


in addition to

$$
m(\lambda)+n(\lambda)=\text { odd integer } \quad(\lambda=1,2,3, \cdots) .
$$

Since $A(z)$ is a meromorphic function of Grommer type, Theorem 1 is applicable. By assertions II, III and IV of this Theorem, we conclude that all the zeros $\zeta_{\lambda}(\lambda)$ of $Q_{\lambda}(z)$ are real and also, in view of (31),

$$
\sum_{j=1}^{d(\lambda)}\left|\zeta_{j}(\lambda)\right|^{-\xi}<\chi
$$

Hence we may apply Lemma $\mathrm{A}$ and find that the three sequences (17) and (18) are uniformly bounded in every disk $\Delta(R)=\{z:|z| \leqq$ $R\}$. Consequently, given an infinite sequence $\Lambda$ of positive increasing integers, it is possible, by the selection principle and Vitali's theorem, to extract a subsequence $\Lambda^{\prime} \subset \Lambda$ such that, as $\lambda \rightarrow \infty, \lambda \in \Lambda^{\prime}$, we have

$$
\begin{aligned}
Q_{\lambda}(z) \exp \left(W_{\lambda}(z)\right) & \rightarrow H(z), \\
P_{\lambda}(z) \exp \left(W_{\lambda}^{\prime}(z)\right) & \rightarrow G(z), \\
W_{\lambda}(z)-W_{\lambda}(z) & \rightarrow \tilde{\Omega}(z),
\end{aligned}
$$

uniformly in $\Delta(R)$.

In view of the arbitrary character of $R$, it is clear that $H(z), G(z)$ are entire functions and $\widetilde{\Omega}(z)$ a polynomial of degree $\leqq s$. Moreover, by (19), these functions satisfy the identity

$$
A(z) H(z)-G(z) \exp (\tilde{\Omega}(z)) \equiv 0,
$$

with

$$
H(0)=1, G(0)=A(0) H(0)=a_{0}, \tilde{\Omega}(0)=0 .
$$

We now prove

Lemma 10.1. Let $h(z)$ be the Weierstrass product in (33). Then

$$
H(z) \equiv h(z) .
$$

Proof. Notice first that

$$
H\left(\boldsymbol{\beta}_{j}\right)=0 \quad(j=1,2,3, \cdots) .
$$

If this were not true, $A(z) H(z)=G(z) \exp (\Omega(z))$ would have poles and this contradicts the fact that $G(z)$ and $\Omega(z)$ are entire. In view of (10.8), $H(z)$ does not vanish identically and consequently we can find arbitrarily large values of $R$ such that

$$
H\left(R e^{i \theta}\right) \neq 0, \quad h\left(R e^{i \theta}\right) \neq 0 \quad(0 \leqq \theta<2 \pi) .
$$


Let $R=R^{*}$ be a value satisfying $(10.11)$ and let there be exactly $p$ zeros of $h(z)$ (that is $p$ points $\beta$ ) in the disk $\Delta\left(R^{*}\right)$.

By Theorem $1, A_{m n}(x) \equiv Q_{\lambda}(x) A_{m}^{(n)}(m=m(\lambda), n=n(\lambda))$ cannot have more than $p$ simple zeros in the disk $\Delta\left(R^{*}\right)$.

Hence (10.11), the uniform convergence in (10.4) and Rouche's theorem show that $H(z)$ cannot have more than $p$ zeros in $\Delta\left(R^{*}\right)$. Returning to (10.10) we see that the zeros of $h(z)$ coincide exactly (their positions as well as multiplicities) with the zeros of $H(z)$. Consequently

$$
H(z)=h(z) e^{\psi(z)}
$$

where $\psi(z)$ is an entire function.

Our next aim is to prove that $\psi(z)$ is a polynomial of degree $\leqq s$.

If $\xi \neq$ integer, this is almost immediate because by (10.4), (10.3) and (8.3),

$$
|H(z)| \leqq \exp \left(\boldsymbol{K}_{2}(s, \xi) \chi|z|^{\xi}\right)
$$

Hence, as in the proof of (3.7),

$$
m\left(r, \exp (\psi(z)) \leqq m(r, H)+m(r, 1 / h)=o\left(r^{s+1}\right) \quad(r \rightarrow \infty) .\right.
$$

This is only possible if $\psi(z)$ is a polynomial of degree $\leqq s$.

If $\xi=s+1,(10.13)$ merely yields $m(r, H)=0\left(r^{s+1}\right)(r \rightarrow \infty)$, and the following more delicate estimates are required to show that, in fact,

$$
m(r, H)=o\left(r^{s+1}\right) \quad(r \rightarrow \infty) .
$$

Given $\epsilon>0$, determine $R_{1}>0$ such that

$$
\frac{2}{R_{1}{ }^{\xi}}+\sum_{\left|\beta_{j}\right| \geqq R_{1}} \frac{1}{\left|\beta_{j}\right|^{\xi}}<\epsilon,
$$

and notice that, by assertion III of Theorem 1, this implies

$$
\sum_{\left|\zeta_{j}(\lambda)\right| \geqq R_{1}} \frac{1}{\left|\zeta_{j}(\lambda)\right|^{\xi}}<\epsilon .
$$

We also have, by (10.3),

$$
\sum_{\left|\zeta_{j}(\lambda)\right|<R_{1}} \frac{1}{\left|\zeta_{j}(\lambda)\right|^{s}} \leqq R_{1} \sum_{\left|\zeta_{j}(\lambda)\right|<R_{1}} \frac{1}{\left|\zeta_{j}(\lambda)\right|^{\xi}}<R_{1} \chi
$$

Combining (10.16), (10.17) and (8.8) we find

$$
\log \left|Q_{\lambda}(z) \exp \left(W_{\lambda}(z)\right)\right| \leqq K_{1}(s) R_{1} \chi|z|^{s}+K_{1}(s) \epsilon|z|^{s+1} \quad(s \geqq 1) .
$$


In view of (10.4) the left-hand side of (10.18) may be replaced by $\log |H(z)|$ and (10.15) follows immediately if $s \geqq 1$. For $s=0$, the proof of (10.15) requires minor modifications which will be left to the reader. Hence (10.14) is always valid and the degree of $\psi(z)$ can never exceeds.

Notice now that the analytic functions

$$
\log \left\{Q_{\lambda}(z) \exp \left(W_{\lambda}(z)\right)\right\}, \quad \log h(z) \quad(\log h(0)=0)
$$

are regular for $|z| \leqq \gamma<\min _{j}\left\{\left|\beta_{j}\right|\right\}$. Consider the Taylor expansions, convergent for $|z| \leqq \gamma$, of the functions (10.19); by (10.4), (10.12) and the definition of $E(u, s)$, we find

$$
\begin{aligned}
& -\frac{z^{s+1}}{s+1} \sum_{j=1}^{d(\lambda)} \frac{1}{\left(\zeta_{j}(\lambda)\right)^{s+1}}-\frac{z^{s+2}}{s+2} \sum_{j=1}^{d(\lambda)} \frac{1}{\left\{\zeta_{j}(\lambda)\right\}^{s+2}}-\cdots \\
& \rightarrow-\frac{z^{s+1}}{s+1} \sum_{j=1}^{\infty} \frac{1}{\beta_{j}^{s+1}}-\frac{z^{s+2}}{s+2} \sum_{j=1}^{\infty} \frac{1}{\beta_{j}^{s+2}}-\cdots+\psi(z)
\end{aligned}
$$

where the convergence is uniform in the disk $|z| \leqq \gamma$. Since $\psi(z)$ is a polynomial of degree $\leqq s,(10.20)$ and Weierstrass' double series theorem imply $\psi(z) \equiv 0$. This completes the proof of Lemma 10.1.

We have thus shown that the limit function $H(z)$ is independent of the choice of $\Lambda$. Hence the restriction $\lambda \in \Lambda^{\prime} \subset \Lambda$ may be omitted and we have proved (36) under the hypotheses (10.1) and (10.2). The condition that $m+n$ is odd is only needed to ascertain the validity of assertion IV of Theorem 1. For functions of Stieltjes type it becomes unnecessary.

Now (10.7), (36) and (34) (which is already proved) yield

$$
A(z) h(z)=G(z) \exp (\tilde{\Omega}(z))=a_{0} g(z) \exp (\Omega(z)),
$$

and therefore, taking (10.5) into account, we find

$$
P_{\lambda}(z) \exp \left(W_{\lambda}(z)\right) \rightarrow G(z)=a_{0} g(z) \exp (\Omega(z)-\tilde{\Omega}(z)) .
$$

By Lemma $\mathrm{A}$, some neighborhood of the origin contains no zeros of $P_{\lambda}(z)\left(\lambda>\lambda_{0}\right)$. This enables us to take logarithms and to deduce from $(10.22)$

$$
\log \left(\frac{P_{\lambda}(z)}{a_{0}}\right)+W_{\lambda}^{\prime}(z) \rightarrow \log g(z)+\Omega(z)-\tilde{\Omega}(z),
$$

and hence 


$$
\begin{gathered}
-\frac{z^{s+1}}{s+1} \sum_{j=1}^{\delta(\lambda)} \frac{1}{\left(\omega_{j}(\lambda)\right)^{s+1}}-\frac{z^{s+2}}{s+2} \sum_{j=1}^{\delta(\lambda)} \frac{1}{\left\{\omega_{j}(\lambda)\right\}^{s+2}}-\cdots \\
\rightarrow-\frac{z^{s+1}}{s+1} \sum_{j=1}^{\infty} \frac{1}{\alpha_{j}^{s+1}}-\frac{z^{s+2}}{s+1} \sum_{j=1}^{\infty} \frac{1}{\alpha_{j}^{s+2}}-\cdots+\Omega(z)-\tilde{\Omega}(z) .
\end{gathered}
$$

The latter relation is entirely analogous to $(10.20)$ and, by the same arguments, leads to $\Omega(z) \equiv \Omega(z)$. Using this identity in (10.22) we obtain (37), and using it in (10.6) we obtain (38).

This completes the proof of Theorem 2 with the only restrictions (10.1).

11. Proof of assertions III and IV of Theorem 2 in the case $m \leqq$ $n-1$. We continue to assume $m(\lambda)+n(\lambda)=$ odd integer $(\lambda=$ $1,2,3, \cdots)$, and reverse the sign of $(10.1)$. Hence, by assumption

$$
m(\lambda) \leqq n(\lambda)-1 ; m(\lambda) \rightarrow \infty(\lambda \rightarrow \infty) .
$$

Put $\nu(\lambda)=m(\lambda), \mu(\lambda)=n(\lambda)-2$, so that

$$
\begin{gathered}
\mu(\lambda)+\nu(\lambda)=\text { odd integer, } \\
\mu(\lambda) \geqq \nu(\lambda)-1,
\end{gathered}
$$

and by (5)

$$
\mu(\lambda) \rightarrow \infty, \nu(\lambda) \rightarrow \infty \quad(\lambda \rightarrow \infty) .
$$

By Lemma 6.1, $D(z)$ is of Grommer type and its poles coincide exactly with the zeros of $A(z)$. Assertions I and II of Theorem 2 [already proved] show that

$$
D(z)=d_{0} \frac{h^{*}(z)}{g(z)} \quad\left(h^{*}(0)=1\right)
$$

where $h^{*}(z)$ is an entire function.

Consider the normalized Padé polynomials $T(z)$ and $S(z)$ of $D(z)$. More precisely, write

$$
T_{\mu \nu}(z)=T_{\lambda}(z), S_{\mu \nu}(z)=S_{\lambda}(z) \quad(\mu=\mu(\lambda), \nu=\nu(\lambda)),
$$

where

$$
\begin{array}{cc}
\text { degree } T_{\lambda}(z) \leqq \nu(\lambda), & T_{\lambda}(0)=1, \\
\text { degree } S_{\lambda}(z) \leqq \mu(\lambda), & S_{\lambda}(0)=d_{0} \\
D(z) T_{\lambda}(z)-S_{\lambda}(z)=z^{\mu+\nu+1} \delta(z) .
\end{array}
$$


From the latter relation and (6.1) we deduce

$$
\begin{aligned}
\frac{T_{\lambda}(z)}{A(z)}-\left(\left(b_{0}+b_{1} z\right) T_{\lambda}(z)-z^{2} S_{\lambda}(z)\right) & =z^{\mu+\nu+3} \delta(z) \\
& =z^{m+n+1} \delta(z) .
\end{aligned}
$$

In view of (11.3), the polynomial

$$
\left(b_{0}+b_{1} z\right) T_{\lambda}(z)-z^{2} S_{\lambda}(z)=b_{0} Q_{\lambda}{ }^{*}(z) \quad\left(Q_{\lambda}{ }^{*}(0)=1\right)
$$

is of degree not greater than $\mu(\lambda)+2=n(\lambda)$.

Clearly (11.7) is equivalent to

$$
A(z) Q_{\lambda}^{*}(z)-a_{0} T_{\lambda}(z)=z^{m+n+1} \delta(z)
$$

and an obvious use of the uniqueness of the Padé polynomials leads at once to the identities

$$
\begin{array}{ll}
Q_{\lambda}{ }^{*}(z) \equiv Q_{\lambda}(z) & (\lambda=1,2,3, \cdots), \\
a_{0} T_{\lambda}(z) \equiv P_{\lambda}(z) & (\lambda=1,2,3, \cdots),
\end{array}
$$

An inspection of (11.2), (11.3) and (11.4) indicates that the results of $\$ 10$ will be readily applicable to the Padé polynomials $T_{\lambda}(z)$ and $S_{\lambda}(z)$ of the function $D(z)$ (of Grommer type). This becomes obvious if we remark that the polynomials $T_{\lambda}(z)$ (or equivalently the polynomials $P_{\lambda}(z)$ ) satisfy the Lindwart-Pólya condition in the form

$$
\sum_{j=1}^{\delta(\lambda)} \frac{1}{\left|\omega_{j}(\lambda)\right|^{\xi}}<\sum \frac{1}{\left|\alpha_{j}\right|^{\xi}} .
$$

By the results of $\S 10$, we now have, as $\lambda \rightarrow \infty$,

$$
\begin{aligned}
& T_{\lambda} \exp \left(W_{\lambda}^{\prime}(z)\right)=\frac{1}{a_{0}} P_{\lambda}(z) \exp \left(W_{\lambda}(z)\right) \rightarrow g(z), \\
& S_{\lambda} \exp \left(W_{\lambda}^{\prime}(z)\right) \rightarrow d_{0} h^{*}(z),
\end{aligned}
$$

uniformly on every compact set $D$. Hence, in view of (11.8), (11.10), (11.5) and (6.1)

$$
\begin{aligned}
& b_{0} Q_{\lambda}(z) \exp \left(W_{\lambda}(z)\right) \rightarrow\left(b_{0}+b_{1} z\right) g(z)-z^{2} d_{0} h^{*}(z) \\
& =\frac{g(z)}{A(z)}=b_{0} h(z) e^{-\Omega(z)} \quad(\lambda \rightarrow \infty) .
\end{aligned}
$$


The latter relation shows that there will exist suitable bounds $\rho^{*}>0, \lambda_{0}$, such that the polynomials $Q_{\lambda}(z)$ will have no zeros for $|z| \leqq \rho^{*}, \lambda>\lambda_{0}$. The first $s+1$ coefficients of the expansion in power series of $\log \left(Q_{\lambda}(z) \exp \left(w_{\lambda}(z)\right)\right.$ are

$$
0,-\frac{\sigma_{k}(\lambda)}{k}+\frac{\tau_{k}(\lambda)}{k} \quad(k=1,2, \cdots, s),
$$

and hence, by (11.13) and Weierstrass' double series theorem,

$$
-W_{\lambda}(z)+W_{\lambda}^{\prime}(z) \rightarrow-\Omega(z) \quad(\lambda \rightarrow \infty) .
$$

Relations such as (36), (37) and (38) follow immediately from (11.12), (11.13) and (11.14). This completes, for functions of Grommer type, the study of the convergence of the Pade polynomials restricted by the condition (11.1). For functions of Stieltjes type the proof is analogous and uses Lemma 6.2 instead of Lemma 6.1. The details are obvious and will be left to the reader.

An inspection of (10.1) and (11.1) shows that it is unnecessary to specify inequalities between $m(\lambda)$ and $n(\lambda)$ and we are thus led to the general symmetrical form of Theorem 2.

\section{REFERENCES}

1. R. J. Arms and A. Edrei, The Padé tables and continued fractions generated by totally positive sequences, Mathematical Essays dedicated to A. J. Macintyre, pp. 1-21, Ohio University Press, Athens (Ohio), 1970.

2. G. A. Baker, Jr., The theory and application of the Padé approximant method, Adv. Theoret. Phys. 1 (1965), 1-58.

3. A. Edrei, Sur les déterminants récurrents et les singularités d'une fonction donnée par son développement de Taylor, Compositio Math. 7 (1939), 20-88.

4. - The Padé table of functions having a finite number of essential singularities, to appear in Pacific J. Math.

5. - Convergence of the complete Padé tables of trigonometric functions, to appear in J. Approximation Theory.

6. N. R. Franzen, Some convergence results for Padé approximants, J. Approximation Theory 6 (1972), 254-263.

7. - Convergence of Padé approximants for a certain class of meromorphic functions, J. Approximation Theory 6 (1972), 264-271.

8. J. Grommer, Ganze transzendente Funktionen mit lauter reellen Nullstellen, Journal f.r.u. angew. Math. 144 (1914), 114-165.

9. E. Lindwart and G. Pólya, Über einen Zusammenhang zwischen der Konvergenz von Polynomfolgen und der Verteilung ihrer Wurzeln, Rendiconti di Palermo 37 (1914), 297-304.

10. R. Nevanlinna, Eindeutige analytische Funktionen, 2nd ed., Berlin, 1953.

11. G. Szegö, Orthogonal polynomials, Amer. Math. Soc. Colloquium Publications, New York, 1967.

12. E. C. Titchmarsh, The Theory of functions, 2nd ed., Oxford, 1950.

Syracuse University, Syracuse, N.Y. 13210 